CLINICAL STUDY

\title{
Endocrine health conditions in adult survivors of childhood cancer: the need for specialized adult-focused follow-up clinics
}

\author{
E Brignardello, F Felicetti ${ }^{1}$, A Castiglione $^{2}, \mathrm{P} \mathrm{Chiabotto}^{3}$, A Corrias $^{3}$, F Fagioli $^{4}$, G Ciccone $^{2}$ and G Boccuzzi ${ }^{1}$ \\ Transition Unit for Childhood Cancer Survivors, San Giovanni Battista Hospital, Via Cherasco 15, 10126 Turin, Italy, ${ }^{1}$ Oncological Endocrinology, \\ University of Turin, Turin, Italy, ${ }^{2}$ Unit of Clinical Epidemiology, San Giovanni Battista Hospital, CPO-Piemonte and University of Turin, Turin, Italy, \\ ${ }^{3}$ Pediatric Endocrinology and ${ }^{4}$ Pediatric Hematology/Oncology Unit, Regina Margherita Children's Hospital, Turin, Italy \\ (Correspondence should be addressed to E Brignardello; Email: ebrignardello@molinette.piemonte.it)
}

\begin{abstract}
Background: Survival rates among childhood cancer survivors (CCS) have enormously increased in the last 40 years. However, this improvement has been achieved at the expense of serious late effects that frequently involve the endocrine system.

Aim: To evaluate the cumulative incidence of endocrine diseases in a cohort of long-term CCS.

Materials and methods: We analyzed the clinical data of 310 adults, followed for a median time of 16.0 years after the first cancer diagnosis. The monitoring protocols applied to each patient were personalized on the basis of cancer diagnosis and previous treatments, according to the Children's Oncology Group guidelines.

Results: The cumulative incidence of endocrine late effects steadily increased over time. At the last follow-up visit available, $48.46 \%$ of females and $62.78 \%$ of males were affected by at least one endocrine disease. The most common disorders were gonadal dysfunction, primary hypothyroidism, and $\mathrm{GH}$ deficiency (GHD). The main risk factors for endocrine disease were male sex (hazard ratio $(\mathrm{HR})=1.45,95 \%$ confidence interval $(95 \% \mathrm{CI}) 1.05-1.99)$, radiotherapy $(\mathrm{HR}=1.91,95 \% \mathrm{CI}$ 1.28-2.84), hematopoietic stem cells transplantation (HR $=3.11,95 \%$ CI 2.23-4.34), and older age at cancer diagnosis $(\mathrm{HR}=1.89,95 \%$ CI $1.25-2.85)$. Male sex was associated with a higher risk of gonadal disorders, whereas radiotherapy specifically increased the risk of GHD and thyroid dysfunction.

Conclusions: Endocrine disorders among CCS have a high prevalence and increase over time. Thus, endocrinologists need to cope with an increasing demand for health care in a field that is still little developed and that, in perspective, could also be extended to some selected types of adult cancer survivors.
\end{abstract}

European Journal of Endocrinology 168 465-472

\section{Introduction}

Thanks to continuous improvements in treatment protocols over the last 40 years, the probabilities of recovery for children and adolescents affected by tumors have risen steadily and, taking into consideration all types of childhood tumors, recovery rates are now at around $80 \%$ (1). This means that there is an increasingly higher number of adults who are pediatric cancer survivors among the general population. However, the concept of 'recovery' refers to the primary tumor and it does not take into consideration the risk or presence of pathological alterations due to late toxicity of anticancer therapy.

It has been reported that 30 years after diagnosis of a pediatric tumor, about two thirds of the survivors will have at least one chronic illness related to previous treatments and, in one third of the cases, the pathological alterations will be so serious as to lead to potentially disabling or life-threatening diseases (2).

The most common alterations involve the endocrine system (3, 4, 5), even if psychological disturbances, neurological and cognitive deficits, heart and respiratory diseases are not rare (6). There is also a significant increase in the risk of a second tumor, especially in those subjects who had previously undergone radiotherapy $(7,8)$.

These late effects, the onset of which basically depends on the type of treatment received, may arise a number of years after treatment and recovery from the tumor $(9,10)$. There is, therefore, a widely shared view that so-called childhood cancer survivors (CCS) should be followed and clinically monitored - also in adulthood and ideally throughout the entire life span - in order to 
reduce morbidity and mortality related to the late toxicity of antitumor treatments $(11,12,13)$.

The 'Transition Unit for Childhood Cancer Survivors' is a specialized adult-focused follow-up clinic within the San Giovanni Battista Hospital of Turin (Piedmont, Italy), headed by an endocrinologist and organized with a network of 'committed' specialists. CCS are usually transitioned to this unit when they are aged over 18 years and off-therapy for at least 5 years. The choice to transfer the follow-up from a pediatric to an adult environment follows the trend of international literature $(11,14,15)$ because of the availability of diagnostic services and consultants more suitable to the patient's age. Furthermore, transition into an adult-focused care may have a beneficial effect on the psychological growth and development of the patients, who, in most cases, seem to be heavily dependent on their parents before transition (16). The monitoring protocols applied to each patient are personalized on the basis of risk stratification, that is, to the cancer diagnosis and previous anticancer treatments, according to the Children's Oncology Group (COG) guidelines (17). Our aim is to evaluate retrospectively the cumulative incidence of endocrine diseases in a cohort of longterm CCS and to identify potential risk factors of the most common endocrine late effects.

\section{Materials and methods}

\section{Patients}

We considered all patients referred to the 'Transition Unit for Childhood Cancer Survivors' from November 2001 to March 2012 who satisfied all the following criteria: i) age at diagnosis under 18 years; ii) at least 5 years of survival after the first cancer diagnosis; and iii) at least one visit after the 18 th birthday.

According to the COG guidelines (17), these patients underwent periodical evaluations including physical examinations as well as laboratory and instrumental tests. We collected retrospectively clinical information about the cancer diagnosis, the treatment, and the medical history (relapses, second tumors, late toxicity at endocrine, cardiac, pulmonary levels, etc.) before transition and recorded the onset of endocrine problem or any other late effect of cancer therapy during follow-up.

We considered the following as endocrine problems: GH deficiency (GHD), primary hypothyroidism, central hypothyroidism, spermatogenesis damage, male and female primary hypogonadism, hypogonadotropic hypogonadism, low bone mineral density (BMD), diabetes mellitus, diabetes insipidus, hyperprolactinemia, and central hypoadrenalism.

Because overweight and dyslipidemia are known to be multifactorial, thus not necessarily a late effect of cancer therapy, they were not considered as endocrine problems for the present analyses.
In order to evaluate the representativeness of our cohort, we linked the data of the Childhood Cancer Registry of Piedmont with our cohort of patients. Two hundred and fifty five out of 310 patients of our cohort were present in the Childhood Cancer Registry of Piedmont and represents about $13.2 \%$ of patients eligible resident in Piedmont at the time of diagnosis. When we consider only more recent years (since 1990), the proportion of eligible patients followed by our unit increased to $20.9 \%$.

\section{Measurements of outcomes}

GHD was always diagnosed before transition. Clinical and auxological examinations were performed in all patients every 6 months. In patients with auxological parameters indicating growth failure, GHD was evaluated using standard provocative tests after all other possible causes of growth failure had been ruled out.

GHD patients were not retested after transition, as we are reluctant to treat with $\mathrm{GH}$ replacement therapy adult survivors of childhood cancer. However, insulinlike growth factor 1 levels measured after transition resulted in agreement with the previous diagnosis.

Primary hypothyroidism was defined as elevated TSH levels with or without free thyroxine $\left(\mathrm{fT}_{4}\right) /$ free tri-iodothyronine reduction; patients with reduced $\mathrm{fT}_{4}$ levels and low or normal TSH levels were considered to have central hypothyroidism.

Sex steroid production was evaluated measuring FSH, $\mathrm{LH}$, inhibin B, and testosterone or $17 \beta$-estradiol serum levels. Patients with clinically significant low levels of sex steroids were considered to have hypogonadism, further subclassified as primary or central, depending on gonadotropin levels. In males, fertility was preferably evaluated with semen sample analysis. As CCS are often reluctant to provide semen samples, male patients with FSH levels $>10 \mathrm{IU} / \mathrm{l}$ and/or inhibin $\mathrm{B}<100 \mathrm{pg} / \mathrm{ml}$ were also considered to have spermatogenesis damage.

We evaluated BMD in lumbar spine or proximal femur using dual-energy X-ray absorptiometry. As index of BMD, we chose Z-score, as T-score is not suitable for females before menopause and for males younger than age 50 years (18). According to other studies describing bone deficits in $\operatorname{CCS}(18,19)$, the cutoff used to identify low BMD is a sex- and age-specific Z-score $<-1.0$.

Patients at risk for adrenal insufficiency were screened by morning cortisol and ACTH basal levels. If morning cortisol and/or ACTH levels were abnormal, as well as in the presence of risk factors and symptoms suggesting adrenal insufficiency, patients were submitted to standard provocative testing to confirm or exclude this diagnosis. Diabetes mellitus was diagnosed by fasting plasma glucose (FPG) concentration, i.e. FPG $\geq 126 \mathrm{mg} / \mathrm{dl}(7.0 \mathrm{mmol} / \mathrm{l})$ confirmed by two or more repeated tests. 
Table 1 Patients and treatment characteristics.

\begin{tabular}{|c|c|c|c|}
\hline & $\begin{array}{l}\text { All } \\
\text { survivors } \\
(n(\%))\end{array}$ & $\begin{array}{c}\text { Survivors with } \\
\text { endocrine disorders } \\
(n(\%))\end{array}$ & $\begin{array}{l}\text { Survivors without } \\
\text { endocrine disorders } \\
(n(\%))\end{array}$ \\
\hline Total & 310 & 176 & 134 \\
\hline \multicolumn{4}{|l|}{ Gender } \\
\hline Female & $130(41.94)$ & $63(35.80)$ & $67(50.00)$ \\
\hline Male & $180(58.06)$ & $113(64.20)$ & $67(50.00)$ \\
\hline \multicolumn{4}{|l|}{ Age at first cancer diagnosis (years) } \\
\hline $0-4$ & $64(20.65)$ & $37(21.02)$ & $27(20.15)$ \\
\hline $5-9$ & $91(29.35)$ & $52(29.55)$ & $39(29.10)$ \\
\hline$\geq 10$ & $155(50.00)$ & $87(49.43)$ & $68(50.75)$ \\
\hline \multicolumn{4}{|l|}{ Age (years) } \\
\hline Age at first cancer diagnosis (years) ${ }^{a}$ & $9.97(7.29)$ & $9.89(7.01)$ & $10.31(7.59)$ \\
\hline Age at the last survivor visit (years) & $24.89(7.40)$ & $25.31(8.04)$ & $24.33(7.68)$ \\
\hline $\begin{array}{l}\text { Years between diagnosis and last } \\
\text { survivor visit }\end{array}$ & $16.09(9.51)$ & $16.38(9.89)$ & $15.26(8.84)$ \\
\hline \multicolumn{4}{|l|}{ Period of diagnosis } \\
\hline Before 1990 & $73(23.55)$ & $46(26.14)$ & $27(20.15)$ \\
\hline 1990-1999 & $171(55.16)$ & $96(54.55)$ & 75 (55.97) \\
\hline After 2000 & $66(21.29)$ & 34 (19.32) & $32(23.88)$ \\
\hline \multicolumn{4}{|l|}{ Cancer diagnosis (first diagnosis) } \\
\hline Hematological malignancies & $209(67.42)$ & $105(59.66)$ & $104(77.61)$ \\
\hline Acute lymphoblastic leukemia & $107(34.52)$ & $51(28.98)$ & $56(41.79)$ \\
\hline Hodgkin lymphoma & $58(18.71)$ & $36(20.45)$ & $22(16.42)$ \\
\hline Non-Hodgkin lymphoma & $25(8.06)$ & $6(3.41)$ & $19(14.18)$ \\
\hline Acute myeloblastic leukemia & $19(6.13)$ & $12(6.82)$ & $7(5.22)$ \\
\hline Brain tumors & $49(15.81)$ & $41(23.30)$ & $8(5.97)$ \\
\hline Sarcomas & $33(10.65)$ & $18(10.23)$ & $15(11.19)$ \\
\hline Others & $19(6.13)$ & $12(6.82)$ & $7(5.22)$ \\
\hline \multicolumn{4}{|l|}{ Relapse } \\
\hline None & $264(85.16)$ & $140(79.55)$ & $124(92.54)$ \\
\hline Yes & $46(14.84)$ & $36(20.45)$ & $10(7.46)$ \\
\hline \multicolumn{4}{|l|}{ Treatment modality } \\
\hline Any radiation & $199(64.19)$ & $140(79.55)$ & $59(44.03)$ \\
\hline Total body irradiation & 40 (12.90) & $38(21.59)$ & $2(1.49)$ \\
\hline Cranial irradiation & $74(23.87)$ & 46 (26.14) & $28(20.90)$ \\
\hline Chemotherapy & $294(94.84)$ & $168(95.45)$ & $126(94.03)$ \\
\hline HSCT & $74(23.87)$ & $64(36.36)$ & $10(7.46)$ \\
\hline Surgery & $115(37.10)$ & $77(43.75)$ & $38(28.36)$ \\
\hline
\end{tabular}

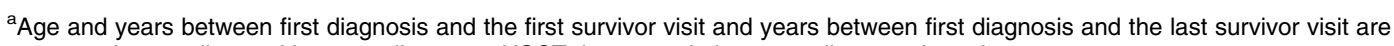
expressed as median and interquartile range. HSCT, hematopoietic stem cells transplantation.

\section{Statistical analysis}

The characteristics of the patients were described using medians and interquartile ranges for the continuous variables. Percentage frequencies were used for the categorical variables. First, we considered the 18th birthday as the time at entry into the survivor program, in order to estimate any endocrine problem cumulative incidence by gender or by cancer diagnosis during clinical follow-up.

Moreover, we also estimated the cumulative incidence of any endocrine disorder since the year of the first diagnosis. Factors potentially influencing the occurrence of endocrine diseases considered in the analysis were sex, age at first cancer diagnosis, cancer diagnosis, and type of treatment. Because almost all patients were exposed to chemotherapy, we could not evaluate this exposure as a potential risk factor. A Cox proportional hazard model was employed to estimate the crude and adjusted hazard ratios (HRs) and 95\% confidence intervals $(95 \%$ CIs) for a set of potential, pre-defined, risk factors and the occurrence of any endocrine conditions and for specific diseases (gonadal disorders, thyroid disorders, GHD, and low BMD). In the Cox models, we considered as time at risk the elapsed time from the date of the first cancer diagnosis until the date of diagnosis of each endocrine disorder.

\section{Results}

Demographic and clinical characteristics of the patients were described in Table 1 . Primary tumors were mainly hematological malignancies $(67.42 \%)$, brain tumors (15.81\%), or sarcomas (10.65\%). Almost all the patients $(94.84 \%)$ had undergone chemotherapy, while radiotherapy and surgery had been carried out in 64.19 and $37.10 \%$ of cases respectively. In most 


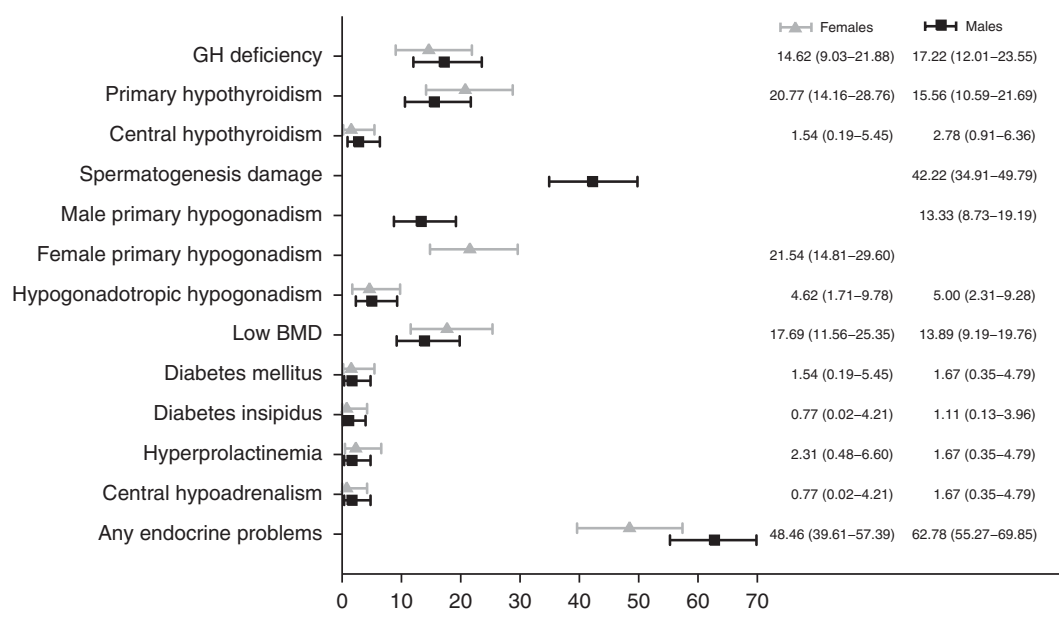

Figure 1 Prevalence of endocrine disorders at the last follow-up visit, by gender. cases, there had been various combinations of these treatments. Moreover, 74 (23.87\%) patients had undergone hematopoietic stem cell transplantation (HSCT). Median age at diagnosis of the first tumor was 10.0 years; median age at the last follow-up visit was 24.89 years. The median length of follow-up since childhood cancer diagnosis was 16.09 years.

We documented the occurrence of at least one endocrine disease in more than $50 \%$ of CCS, $48.46 \%$ in female and $62.78 \%$ in male CCS (Fig. 1). The most frequent specific endocrine disorders were gonadal dysfunction, primary hypothyroidism, and GHD.

GHD was found in 50 CCS (16.13\%); 33 of them had a previous diagnosis of CNS tumor, whereas 17 were cured for hematological malignancies. In almost all cases $(92.00 \%)$, the GHD patients had received radiation therapy involving the head, at doses ranging between 12 and $64 \mathrm{~Gy}$. Besides, 25 patients (18 males and seven females) received GH replacement therapy during childhood, whereas the other 25 (13 males and 12 females) refused, due to their parents' concerns about the risk of recurrence or second malignant neoplasms, with adult short stature as the main consequence.

As far as thyroid late effects, primary hypothyroidism was diagnosed in 55 patients $(17.74 \%)$, whereas seven patients showed central hypothyroidism. Moreover, 18 patients developed a papillary thyroid carcinoma as second neoplasm, which in 15 cases arose after radiation therapy involving the neck. This last result will not be discussed any further in this paper, as it will be the subject of another study.

Primary hypogonadism was found in $13.33 \%$ males and $21.54 \%$ females. Moreover, 76 males $(42.22 \%)$ were considered to have spermatogenesis damage, which in about $40 \%$ of cases was confirmed by semen analysis.

Low BMD (Z-score <-1.0) was found in 48 CCS ( 25 males and 23 females), 24 of them showing a Z-score $<-2.0$.

Type 1 and type 2 diabetes mellitus were found in one and four patients respectively. A low prevalence of hyperprolactinemia (six patients), central hypoadrenalism (four patients), and diabetes insipidus (three patients) was found in our cohort. The cumulative incidence of endocrine late effects steadily increased over time from $24.03 \%$ in males and $26.26 \%$ in females at the 18 th birthday to 72.02 and $50.06 \%$ respectively after 12 years of follow-up (Fig. 2A).

Five years after cancer diagnosis, the cumulative incidence of endocrine disorders was $18.60 \%(95 \%$ CI $12.88-26.46)$ in females and $11.17 \%$ (95\% CI 7.36-16.78) in males, whereas after 10 years, it was $31.07 \%$ (95\% CI $23.73-40.01$ ) and $34.04 \%$ (95\% CI 27.55-41.56) respectively (Fig. 2B).

The risk to develop endocrine disorders was higher for patients with brain tumor than for any other diagnosis (Fig. 3A). As a group, hematological malignancies had a lower risk of developing endocrine diseases but showed a high variability between different diagnoses (Fig. 3B). The crude and adjusted association between patient/treatment characteristics and specific endocrine late effects are shown in Tables 2 and 3 respectively.

Gender and age at first diagnosis were associated with the risk of any endocrine disorders (Tables 2 and 3 ). In detail, the risk is higher in males ( $\mathrm{HR}=1.45,95 \%$ CI 1.05-2.00) and in patients with cancer diagnosed after the age of 10 years (HR $=1.89,95 \%$ CI $1.25-2.85$ ).

Radiation therapy and HSCT increased the risk of any endocrine diseases by two and three times respectively $(\mathrm{HR}=1.91,95 \%$ CI $1.28-2.84$; $\mathrm{HR}=3.11$, 95\% CI 2.23-4.34). The effects of these exposures were confirmed when we considered specific endocrine problems.

\section{Discussion}

Our data indicate that, 16 years after the diagnosis of a childhood cancer, more than one half of survivors suffered or are suffering from at least one endocrine late effect. This result, which agrees with previous reports 

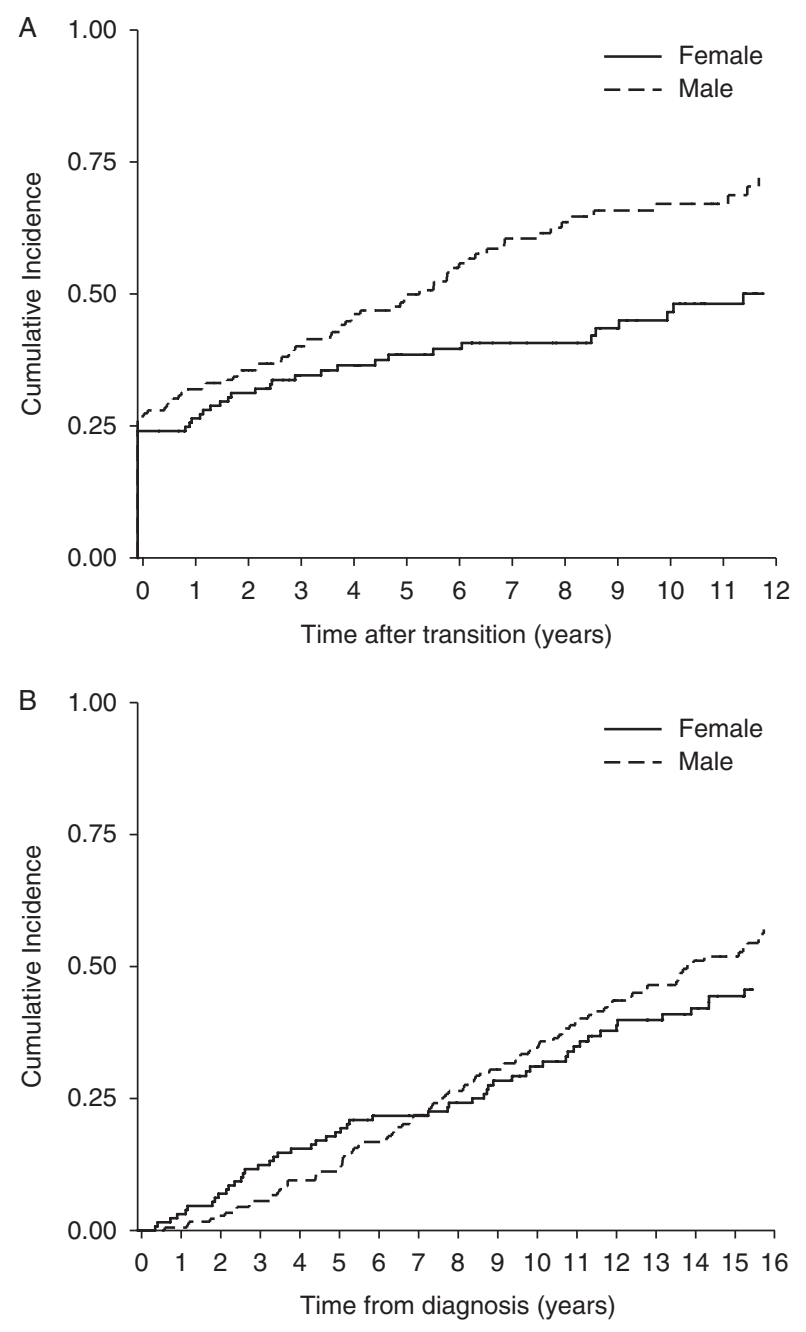

Figure 2 Cumulative incidence of any endocrine disorders after transition $(A)$ and after diagnosis $(B)$ by gender.

$(3,20,21)$, is doubtless alarming especially in a population that - in many countries - still does not receive an adequate follow-up. Anyway, it should be considered that about $50 \%$ of our patients were treated before 1995. In the last 20 years, there have been considerable improvements in the treatment protocols for childhood cancer so that an effective cure rate can now be obtained with substantially fewer late effects. However, it cannot be excluded that patients who were treated more recently will survive in a larger proportion and may develop a different array of long-term chronic complications; therefore, they also need to be followed.

The cumulative incidence of endocrine disorders was higher in males than in females. This result mainly depends on spermatogenesis damage, which is a common condition in male CCS.

The development of endocrine late effects was strongly associated with HSCT and radiation therapy. We previously reported that the use of total body irradiation (TBI) as a conditioning regimen is associated with a worse endocrine outcome in patients submitted to HSCT (22). Here, we show that HSCT, whatever conditioning regimen was used, is associated with a significantly higher risk of endocrine damage, especially of gonadal dysfunction and low BMD. That is not surprising, as HSCT usually requires heavy treatment with gonadotoxic agents and is often performed at relapse; consequently the cumulative exposure to chemotherapy, in these patients, is higher. As far as BMD, the strong association between HSCT and osteopenia is likely caused by the interaction of multiple risk factors related to HSCT (hypogonadism, GHD, decreased physical activity, and intensive chemotherapy including the use of corticosteroids and antimetabolites at high doses) $(23,24)$.

Radiation therapy was found to be independently associated with GHD and thyroid disorders. There is a well-established relationship between the total radiation dose to pituitary and the development of hormone
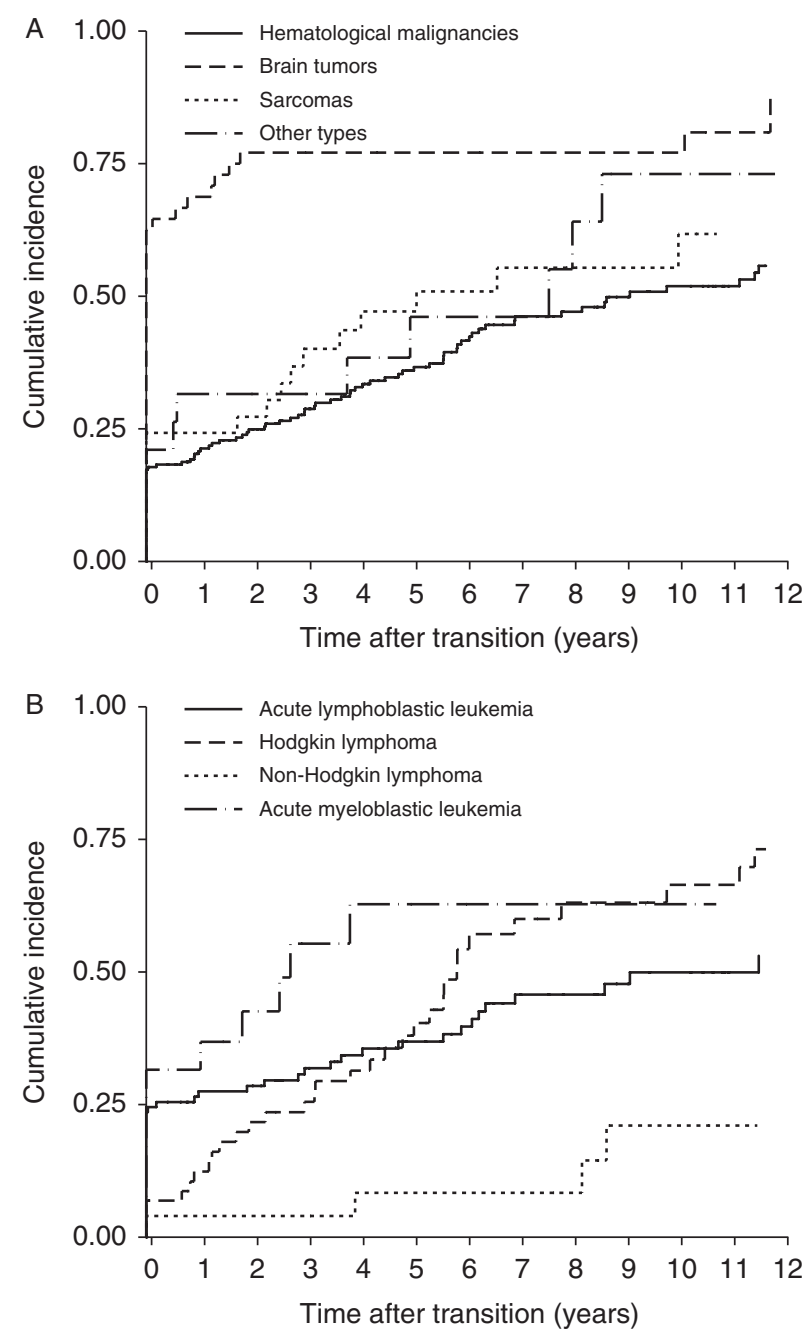

Figure 3 Cumulative incidence of any endocrine disorders by cancer diagnosis $(A)$ and by hematological malignancies type (B). 


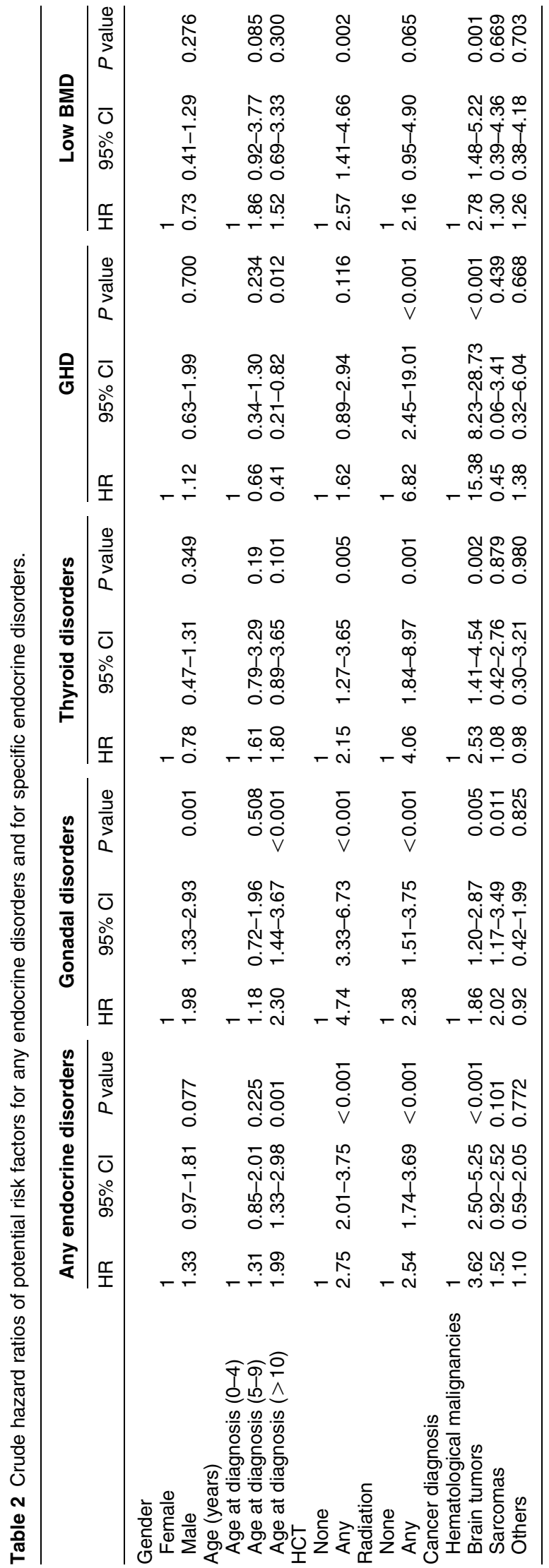

deficits (4). GH is the most sensitive pituitary hormone to radiation therapy, and isolated GHD can be seen after low doses of cranial irradiation as well as after TBI (25). We could not stratify our patients by radiation site or radiation dose. Nevertheless, this result could be explained considering that our cohort includes many patients treated with cranial irradiation, i.e. brain tumors, patients submitted to TBI as conditioning regimen for HSCT, and patients who received radiotherapy to the brain for hematological malignancies.

Radiotherapy also increases the risk of hypothyroidism. Central hypothyroidism is uncommon and usually occurs after cranial irradiation at doses higher than $30 \mathrm{~Gy}$ (26). By contrast, primary hypothyroidism is not rare in CCS who have been treated with external-beam RT involving the thyroid gland $(27,28)$. Indeed, we observed primary hypothyroidism in about $20 \%$ of our patients, mainly after TBI, neck/mantle RT, or craniospinal irradiation for brain tumors.

The risk to develop endocrine late effects was about twofold higher in CCS treated after the age of 10 years. This result confirms a recently reported observation (21) and may be partly due to epidemiological considerations (age distribution of pediatric cancer diagnosis as well as stage at cancer diagnosis). However, the multivariate analysis confirmed this result for gonadal disorders. It has been shown that the ovaries of younger patients are more resistant to the cytotoxic effects of chemotherapy and radiotherapy $(29,30)$. Moreover, testis of younger patients seems to be less sensitive to the damage induced by cancer treatments, but these observations have been questioned $(31,32)$. The risk to develop gonadal disorders was significantly higher in males than in females, likely due to the very high sensitivity of seminiferous tubules to both chemotherapy and radiotherapy (31).

As far as cancer diagnosis is concerned, patients treated for brain tumors have the highest risk to subsequently develop endocrine late effects. Treatment protocols for these patients include - in most cases high-dose radiation therapy that involves the pituitary gland, producing GHD and hypogonadism that also contribute to the development of a low BMD. Among hematological malignancies, non-Hodgkin lymphoma patients show the lowest cumulative incidence of endocrine conditions. By contrast, in survivors of acute myeloblastic leukemia and Hodgkin lymphoma, the risk of endocrine late effects is similar to that observed in brain tumors. Alkylating agents, usually employed at high doses to treat sarcomas, are the main cause for the gonadal dysfunctions frequently observed in these CCS.

\section{Strengths and limitations of the study}

The patients were enrolled at the first access to our Transition Unit. This fact may represent a possible source of selection bias. However, unselected, 


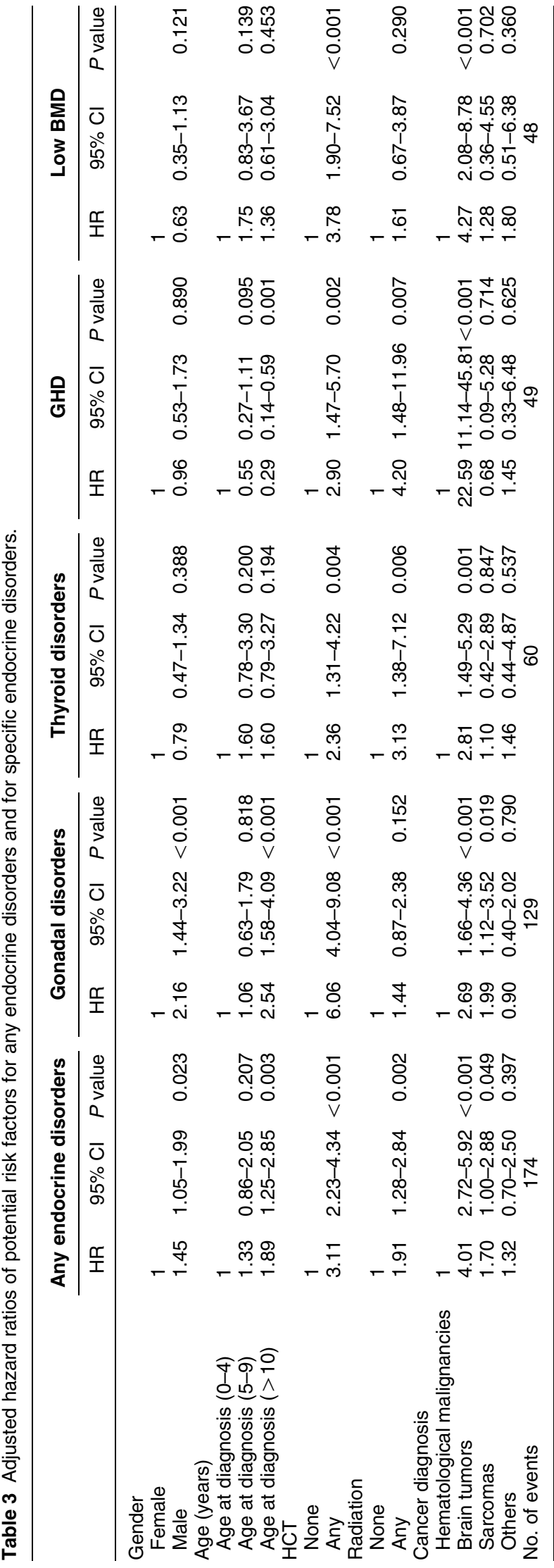

population-based cohorts of adults who were childhood cancer long-term survivors with at least 5 years of survival are quite difficult to assemble and to follow-up.

In all Cox models and when in the estimate of any endocrine cumulative incidence (Fig. 2B), we considered as time at risk the time elapsed from the date of the first cancer diagnosis until the date of endocrine disorder diagnosis, but all data before transition were available only for the selected population of long-term survivors attending the Transition Unit.

\section{Conclusion}

The cumulative incidence of endocrine late effects in CCS is very high and increases over time. Indeed many endocrine disorders were diagnosed after transition; nevertheless, these late effects are often under-diagnosed, probably due to the lack of specialized centers for the long-term follow-up (33).

Thus, the requirement for lifelong monitoring - to unveil and promptly treat the late complications that might arise from their cancer and its therapy - and the high prevalence of endocrine disorders among CCS could entail the need for endocrinologists to cope with an increasing demand for health care in a field that is still little developed and that could, in perspective, also be extended to some selected types of adult cancer.

\section{Declaration of interest}

The authors declare that there is no conflict of interest that could be perceived as prejudicing the impartiality of the research reported.

\section{Funding}

This research did not receive any specific grant from any funding agency in the public, commercial or not-for-profit sector.

\section{Acknowledgements}

The authors would like to thank Fulvio Lazzarato, Carlotta Sacerdote, and the Childhood Cancer Registry of Piedmont for their collaboration.

\section{References}

1 Jemal A, Siegel R, Ward E, Murray T, Xu J, Smigal C \& Thun MJ. Cancer statistics, 2006. CA: A Cancer Journal for Clinicians $2006 \mathbf{5 6}$ 106-130. (doi:10.3322/canjclin.56.2.106)

2 Oeffinger KC, Mertens AC, Sklar CA, Kawashima T, Hudson MM, Meadows AT, Friedman DL, Marina N, Hobbie W, KadanLottick NS et al. Chronic health conditions in adult survivors of childhood cancer. New England Journal of Medicine 2006355 1572-1582. (doi:10.1056/NEJMsa060185)

3 Chemaitilly W \& Sklar CA. Endocrine complications in long-term survivors of childhood cancers. Endocrine-Related Cancer 201017 R141-R159. (doi:10.1677/ERC-10-0002)

4 Nandagopal R, Laverdière C, Mulrooney D, Hudson MM \& Meacham L. Endocrine late effects of childhood cancer therapy: a report from the Children's Oncology Group. Hormone Research 200869 65-74. (doi:10.1159/000111809) 
5 van Dorp W, van Beek RD, Laven JS, Pieters R, de Muinck KeizerSchrama SM \& Van den Heuvel-Eibrink MM. Long-term endocrine side effects of childhood Hodgkin's lymphoma treatment: a review. Human Reproduction Update $2012 \mathbf{1 8}$ 12-28. (doi:10.1093/ humupd/dmrO38)

6 Bhatia S \& Constine LS. Late morbidity after successful treatment of children with cancer. Cancer Journal 200915 174-180. (doi:10.1097/PPO.0b013e3181a58f46)

7 Armstrong GT, Liu W, Leisenring W, Yasui Y, Hammond S, Bhatia S, Neglia JP, Stovall M, Srivastava D \& Robison LL. Occurrence of multiple subsequent neoplasms in long-term survivors of childhood cancer: a report from the Childhood Cancer Survivor Study. Journal of Clinical Oncology 201129 3056-3064. (doi:10.1200/JCO.2011.34.6585)

8 Oeffinger KC \& Bhatia S. Second primary cancers in survivors of childhood cancer. Lancet 2009374 1484-1485. (doi:10.1016/ S0140-6736(09)61885-7)

9 Garwicz S, Anderson H, Olsen JH, Falck Winther J, Sankila R, Langmark F, Tryggvadóttir L, Möller TR \& for the Association of the Nordic Cancer Registries (ANCR) and the Nordic Society for Pediatric Hematology Oncology (NOPHO). Late and very late mortality in 5-year survivors of childhood cancer: changing pattern over four decades. Experience from the Nordic countries. International Journal of Cancer 2012131 1659-1666. (doi:10.1002/ijc.27393)

10 Reulen RC, Frobisher C, Winter DL, Kelly J, Lancashire ER, Stiller CA, Pritchard-Jones K, Jenkinson HC, Hawkins MM \& British Childhood Cancer Survivor Study Steering Group. Longterm risks of subsequent primary neoplasms among survivors of childhood cancer. Journal of the American Medical Association 2011305 2311-2319. (doi:10.1001/jama.2011.747)

11 Henderson TO, Friedman DL \& Meadows AT. Childhood cancer survivors: transition to adult-focused risk-based care. Pediatrics 2010126 128-136. (doi:10.1542/peds.2009-2802)

12 Prasad PK, Bowles $\mathrm{T} \&$ Friedman DL. Is there a role for a specialized follow-up clinic for survivors of pediatric cancer? Cancer Treatment Reviews 201036 372-376. (doi:10.1016/j.ctrv. 2010.02.014)

13 Nathan PC, Hayes-Lattin B, Sisler JJ \& Hudson MM. Critical issues in transition and survivorship for adolescents and young adults with cancers. Cancer 2011117 (suppl 10) 2335-2341. (doi:10.1002/cncr.26042)

14 Freyer DR. Transition of care for young adult survivors of childhood and adolescent cancer: rationale and approaches. Journal of Clinical Oncology 201028 4810-4818. (doi:10.1200/ JCO.2009.23.4278)

15 Gage EA, Pailler M, Zevon MA, Ch'ng J, Groman A, Kelly M, Panagakis C, Wilding GE, Yasko J \& Gruber M. Structuring survivorship care: discipline-specific clinician perspectives. Journal of Cancer Survivorship 20115 217-225. (doi:10.1007/ s11764-011-0174-x)

16 Freyer DR \& Brugieres L. Adolescent and young adult oncology: transition of care. Pediatric Blood \& Cancer 200850 (suppl 5) 1116-1119. (doi:10.1002/pbc.21455)

17 The Children's Oncology Group. Long-term follow-up guidelines for survivors of childhood, adolescent, and young adult cancers 2008 (http://www.survivorshipguidelines.org).

18 Lewiecki EM, Watts NB, McClung MR, Petak SM, Bachrach LK, Shepherd JA, Downs RW Jr \& International Society for Clinical Densitometry. Official positions of the international society for clinical densitometry. Journal of Clinical Endocrinology and Metabolism 2004 89 3651-3655. (doi:10.1210/jc.2004-0124)

19 Kelly J, Damron T, Grant W, Anker C, Holdridge S, Shaw S, Horton J, Cherrick I \& Spadaro J. Cross-sectional study of bone mineral density in adult survivors of solid pediatric cancers. Journal of Pediatric Hematology/Oncology $200527248-253$. (doi:10.1097/01.mph.0000162526.77400.78)

20 Shalitin S, Gal M, Goshen Y, Cohen I, Yaniv I \& Phillip M. Endocrine outcome in long-term survivors of childhood brain tumors. Hormone Research in Paediatrics $2011 \mathbf{7 6}$ 113-122. (doi:10.1159/000327584)
21 Patterson BC, Wasilewski-Masker K, Ryerson AB, Mertens A \& Meacham L. Endocrine health problems detected in 519 patients evaluated in a pediatric cancer survivor program. Journal of Clinical Endocrinology and Metabolism $2012 \quad \mathbf{9 7} \quad 810-818$. (doi:10.1210/jc.2011-2104)

22 Felicetti F, Manicone R, Corrias A, Manieri C, Biasin E, Bini I, Boccuzzi G \& Brignardello E. Endocrine late effects after total body irradiation in patients who received hematopoietic cell transplantation during childhood: a retrospective study from a single institution. Journal of Cancer Research and Clinical Oncology 2011 137 1343-1348. (doi:10.1007/s00432-011-1004-2)

23 Wasilewski-Masker K, Kaste SC, Hudson MM, Esiashvili N, Mattano LA \& Meacham LR. Bone mineral density deficits in survivors of childhood cancer: long-term follow-up guidelines and review of the literature. Pediatrics $2008 \mathbf{1 2 1} 705-713$. (doi:10.1542/peds.2007-1396)

24 Le Meignen M, Auquier P, Barlogis V, Sirvent N, Contet A, Simeoni MC, Galambrun C, Poirée M, Chastagner P, Play B et al. Bone mineral density in adult survivors of childhood acute leukemia: impact of hematopoietic stem cell transplantation and other treatment modalities. Blood $2011 \mathbf{1 1 8} 1481-1489$. (doi:10.1182/blood-2011-01-332866)

25 Darzy KH \& Shalet SM. Pathophysiology of radiation-induced growth hormone deficiency: efficacy and safety of GH replacement. Growth Hormone \& IGF Research 200616 S30-S40. (doi:10.1016/j.ghir.2006.03.002)

26 Schmiegelow M, Feldt-Rasmussen U, Rasmussen AK, Poulsen HS \& Müller J. A population-based study of thyroid function after radiotherapy and chemotherapy for a childhood brain tumor. Journal of Clinical Endocrinology and Metabolism $2003 \mathbf{8 8}$ 136-140. (doi:10.1210/jc.2002-020380)

27 Armstrong GT, Stovall M \& Robison LL. Long-term effects of radiation exposure among adult survivors of childhood cancer: results from the Childhood Cancer Survivor Study. Radiation Research 2010 174 840-850. (doi:10.1667/RR1903.1)

28 Diller L, Chow EJ, Gurney JG, Hudson MM, Kadin-Lottick NS, Kawashima TI, Leisenring WM, Meacham LR, Mertens AC, Mulrooney DA et al. Chronic disease in the Childhood Cancer Survivor Study cohort: a review of published findings. Journal of Clinical Oncology 200927 2339-2355. (doi:10.1200/JCO.2008. 21.1953)

29 Gnaneswaran S, Deans R \& Cohn RJ. Reproductive late effects in female survivors of childhood cancer. Obstetrics and Gynecology International, 2012. Article ID 564794. (doi:10.1155/2012/ 564794)

30 Morgan S, Anderson RA, Gourley C, Wallace WH \& Spears N. How do chemotherapeutic agents damage the ovary? Human Reproduction Update 201218 525-535. (doi:10.1093/humupd/ dms022)

31 van Casteren NJ, van der Linden GH, Hakvoort-Cammel FG, Hählen K, Dohle GR \& van den Heuvel-Eibrink MM. Effect of childhood cancer treatment on fertility markers in adult male long-term survivors. Pediatric Blood E Cancer 200952 108-112. (doi:10.1002/pbc.21780)

32 Green DM, Kawashima T, Stovall M, Leisenring W, Sklar CA, Mertens AC, Donaldson SS, Byrne J \& Robison LL. Fertility of male survivors of childhood cancer: a report from the Childhood Cancer Survivor Study. Journal of Clinical Oncology 201028 332-339. (doi:10.1200/JC0.2009.24.9037)

33 Brabant G, Toogood AA, Shalet SM, Frobisher C, Lancashire ER, Reulen RC, Winter DL \& Hawkins MM. Hypothyroidism following childhood cancer therapy - an under diagnosed complication. International Journal of Cancer $20121301145-1150$. (doi:10.1002/ijc.26086)

Received 18 August 2012

Revised version received 11 December 2012

Accepted 19 December 2012 